Article

\title{
Effects of Disruption Risks on Biorefinery Location Design
}

\author{
Yun Bai ${ }^{1}$, Xiaopeng $\mathrm{Li}^{2}$, Fan Peng ${ }^{3}$, Xin Wang ${ }^{4}$ and Yanfeng Ouyang ${ }^{4, *}$
}

1 Center for Advanced Infrastructure and Transportation, Rutgers, the State University of New Jersey, 100 Brett Road, Piscataway, NJ 08854-8058, USA; E-Mail: yunbai.cait@ rutgers.edu

2 Department of Civil and Environmental Engineering, Mississippi State University, 235L Walker Engineering Building, Starkville, MS 39762-9546, USA; E-Mail: xli@ cee.msstate.edu

3 Operations Research Department, CSX Transportation, Inc., Jacksonville, FL 32202, USA; E-Mail: fan_peng@ csx.com

4 Department of Civil and Environmental Engineering, University of Illinois at Urbana-Champaign, 205 N. Mathews Ave., Urbana, IL 61801, USA; E-Mail: wangxin1@illinois.edu

* Author to whom correspondence should be addressed; E-Mail: yfouyang@illinois.edu; Tel.: +1-217-333-9858; Fax: +1-217-333-1924.

Academic Editor: Calliope Panoutsou

Received: 27 September 2014 / Accepted: 27 January 2015 / Published: 13 February 2015

\begin{abstract}
While ever-growing bio-ethanol production poses considerable challenges to the bioenergy supply chain, the risk of refinery operation disruptions further compromises the efficiency and reliability of the energy supply system. This paper applies discrete and continuous reliable facility location models to the design of reliable bio-ethanol supply chains so that the system can hedge against potential operational disruptions. The discrete model is shown to be suitable for obtaining the exact optimality for small or moderate instances, while the continuous model has superior computational tractability for large-scale applications. The impacts of both site-independent and dependent disruptions (i.e., due to flooding) are analyzed in empirical case study for the State of Illinois (one of the main biomass supply states in the U.S.). The reliable solution is compared with a deterministic solution under the same setting. It is found that refinery disruptions, especially those site-dependent ones, affect both optimal refinery deployment and the supply chain cost. Sensitivity analysis is also conducted to show how refinery failure probability and fixed cost (for building biorefineries) affect optimal supply chain configuration and the total expected system cost.
\end{abstract}


Keywords: biorefinery location; disruption uncertainty; reliable bio-ethanol supply chain network; biomass transportation; discrete facility location model; continuous approximation; Illinois bio-ethanol industry

\section{Introduction}

The U.S. bio-ethanol industry has been experiencing phenomenal growth in recent years. Part of this growth may be attributed to the Energy Policy Act of 2005 and the Energy Independence and Security Act of 2007, which mandate that the annual U.S. ethanol production grow from 9 billion gallons in 2008 to over 36 billion gallons in 2022 [1]. With such a sharp increase in bio-ethanol demand, ethanol manufacturing infrastructure must be significantly expanded to provide sufficient production capacity and ensure overall efficiency and reliability of the bioenergy supply chain.

Biorefinery location decision is a key to the strategic bio-ethanol supply chain design. It directly determines feedstock shipment and final product distribution. Huge capital investment is generally required to build a biorefinery plant, even for one with a moderate size; for example, a mid-sized corn-based biorefinery with a $50 \mathrm{MGY}$ (million gallons per year) capacity costs roughly \$66 million [2]. As large biorefineries become more and more popular, the investment in refinery construction constitutes a major portion of the total supply chain cost. Biomass transportation from farm areas to their nearby refineries is responsible for a large portion of the operation costs, partly because of the low energy density of biomass crops [3]. The transportation cost is usually proportional to the shipment distance. Refinery locations need to be carefully designed to minimize the total cost for the overall bio-ethanol supply chain system (i.e., capital investment and transportation cost).

The biorefinery location problem has been studied only very recently. Tursun et al. [4] proposed a discrete facility location model to optimize the total cost including transporting and processing biomass, delivering ethanol, building and operating biorefineries. They focus on the biofuel industry in Illinois to draw practical implications (e.g., four new refineries would be built by the year 2022). Kang et al. [5] extended this model and adjusted the projected growth of the biofuel industry by incorporating by-products of ethanol production, different types of biomass (both corn and cellulose), and corresponding refineries in a multiyear supply chain design scenario. Bai et al. [6] studied the impact of biofuel supply shipment on transportation congestion and suggested that more refineries are needed with adjusted locations to reduce the public concerns on congestion. Other than strategic planning, system dynamics in biomass logistics operation and management have also been investigated. Eksioglu et al. [7], through a case study about Mississippi, coordinated supply chain design decisions and logistics management decisions to analyze logistical challenges in supplying biomass to a biorefinery. Sokhansanj et al. [8] built an inter-connected discrete events and queuing simulation program to simulate the entire network of material flow from farm to a biorefinery and from collection, storage, to transport operations. The Western Bioenergy Assessment Team at the University of California [9] developed an integrated model for bio-ethanol supply chain design by combining a geo-coded spatial resource inventory and infrastructure assessment system with a cost minimization model. Their efforts helped understand the future development of biofuel industry in the western U.S. GIS 
(Geographic Information System)-based optimization models have also been developed to consider supply of available feedstock and local demand for bio-ethanol [10] and biomass farm gate prices for selecting the least-cost biorefinery locations [11]. Furthermore, Kumar et al. [12], Mahmudi and Flynn [13] both analyzed the cost effectiveness of different biomass transportation modes; the former proposed a multi-criteria assessment methodology that integrates economic, social, environmental, and technical factors to rank alternatives for biomass transportation, while the latter made a statistical comparison between rail and truck transportation modes and found the economical distance for transshipment. With similar statistical tools, Searcy et al. [14] provided the distance criteria for optimal transportation mode selection in the biofuel supply chain. They suggested that the optimal biorefinery location should be close to the source of biomass rather than to the consumption point because of the relatively high cost of moving feedstock.

To the authors' best knowledge, discrete integer programming and continuous models are suitable modeling approaches for network design problems. The refinery location problems essentially fall into the general class of facility location problems, which can be solved to the exact optimal design with proper integer programming models. Integer programming models have been frequently applied to similar system planning problems in various engineering contexts (see "Network and Discrete Location" by Daskin [15]). Another alternative to solve discrete location problems would be to use heuristic algorithms, which however are mainly suitable when exact models have significant computational issues. Continuous approximation (CA) models are often applied to large-scale location problems with continuous settings that are computational intractable for discrete models. With proper treatment (e.g., by using the disc model proposed by Ouyang and Daganzo [16], CA can be applied to discrete settings and yield discrete location design solutions as well. See Newell [17], Langevin et al. [18], and Daganzo [19] for detailed introduction to continuous approximation models.

However, it has been realized recently that the "optimal" design in deterministic settings may also be vulnerable to substantial operational risks from imperfect information and underlying uncertainties (e.g., demand variations and facility disruptions). Supply chain reliability and resilience against such risks have gained increasing attention. Earlier literature examined facility congestion that arises from demand uncertainties and attempted to enhance system availability by providing redundancy [20-24]. After a series of devastating disasters in recent years (e.g., 2005 Hurricane Katrina, 2003 U.S. Northeast blackout, 2008 China and 2009 Haiti Earthquakes), people have recognized the adversary impacts of potential disruptions on the supply side that may be caused by natural disasters, power outages, operational incidents, labor actions or terrorist attacks. As a result, reliable facility location models have been developed to design supply chains that can hedge against the impacts of facility disruptions. Snyder and Daskin [25] proposed a discrete integer programming model for the reliable uncapacitated fixed charge location problem, assuming that facility disruptions occur independently with equal probability. Cui et al. [26] developed not only a generalized discrete model to allow for site-dependent facility disruption probabilities but also an alternative continuous model that significantly improves the computational tractability. Li and Ouyang [27] generalized this continuous model to address spatial correlations that may exist among facility disruptions. Compared with the traditional counterparts, the reliable models have significantly improved system reliability and reduced the expected overall cost across normal and random disruption scenarios. 
Like many other facilities, bio-ethanol refineries are susceptible to disruption hazards such as water scarcity, flooding, routine maintenance, or adverse weather condition $[28,29]$. Once refinery disruption happens, excessive operational cost may occur due to the reallocation of biomass supply to more distant refineries. In addition, huge gasoline price volatility and enormous societal cost (associated with producer or consumer surplus) may be induced [30]. Therefore, in view of refinery disruption uncertainty, bio-ethanol supply chain design questions (e.g., how many and where to build biorefineries, what sizes the refineries should be, and how to distribute feedstock to refineries) need to be addressed systematically to develop economical, reliable and sustainable infrastructure systems that are suitable for satisfying the mandatory future ethanol demand. To the best of our knowledge, only a very recent study by Marufuzzaman et al. [31] considered facility disruptions in biofuel supply chain design. However, this study assumed that once a facility disrupts, the customers originally assigned to this facility is always diverted to a designated perfectly reliable facility, and thus it excludes the possibility of simultaneous disruptions of multiple facilities serving the same customer, which is likely the case when a massive disaster attacks.

This paper focuses on designing bio-ethanol supply chains that not only operate efficiently in the normal (i.e., disruption-free) scenario but also are able to provide sufficient back-ups to minimize cost increase and negative social impacts under refinery disruptions. The studied problem allows for simultaneous refinery disruptions that may force biomass providers to seek more distant refineries or to completely give up supplying the biomass. Both discrete and continuous reliable location models are used to design a reliable bio-ethanol supply chain network for the State of Illinois, where (i) a significant amount of the nation's biomass supply and bioethanol is produced; and (ii) the state has already geared up toward a rapid expansion of bio-ethanol production infrastructure. Our experiments show that the discrete model can solve the reliable design problem almost to exact optimality, while the continuous model yields near-optimum solutions very efficiently (i.e., ideal for large scale application). We also conducted a set of sensitivity analyses to draw insights into how the total supply chain performance and the optimal refinery deployment are affected by parameters such as refinery disruption probabilities and fixed costs of building biorefineries.

The contribution of this paper is two-fold. First, it examines and compares the applicability of two state-of-the-art location design models, with discrete and continuous formulations respectively, on biofuel infrastructure design, considering the critical issue of infrastructure disruptions. Second, from the comprehensive case studies, we obtained a number of planning alternatives under a variety of system settings and revealed significant insights into the relationship between the system setting and the biofuel infrastructure design. Such results provide valuable guidance to overcoming the critical challenge of biofuel infrastructure planning in the State of Illinois, one of the states producing the highest biomass supply in the U.S.

This paper is organized as follows. Section 2 briefly introduces the discrete and continuous reliable facility location models in the bio-ethanol supply chain context. Section 3 describes the refinery location design problem faced by the Illinois bio-ethanol industry. Section 4 presents findings from the case study, including comparison of the discrete and continuous models and results from the sensitivity analyses. Section 5 concludes the paper and discusses future research directions. 


\section{Model Formulation}

This section briefly introduces two reliable refinery facility location models. We will first present a discrete reliable uncapacitated facility location model and a Lagrangian relaxation based solution approach that are developed in [26,32]. This approach generally can solve small to moderate instances to the exact optimality. Then we present a continuum approximation approach in [27] that is suitable for large-scale instances with slow-varying conditions, and can obtain approximated near-optimum solutions within a much shorter time.

\subsection{Discrete Model}

Let $\mathcal{J}=\{0,1, \cdots,|\mathcal{J}|-1\}$ denote the set of candidate locations where refinery facilities can be potentially built to collectively "serve" a set of farms, $\mathcal{I}=\{0,1, \cdots,|\mathcal{I}|-1\}$, where farm $i \in \mathcal{I}$ produces $\lambda_{i}$ bushels of corn each year for biofuel supply. Building a refinery at location $j \in \mathcal{J}$ requires a prorated annual construction cost $f_{j}$; transporting each bushel of corn from farm $i$ to refinery $j$ imposes a yearly transportation cost $d_{i j}$.

A refinery, if built at candidate location $j$, is subject to a site-dependent disruption probability $q_{j}$. Assume refinery disruptions are independent and in each disruption scenario (i.e., the pattern/combination of refinery disruptions), only functioning refineries can produce bio-ethanol; the failed ones completely halt all their production lines. To hedge against such disruption risks, each farm $i$ is assigned to a subset of at most $R \geq 1$ refineries. The refineries in this subset are labeled with rank $r=0,1, \cdots, R-1$, which represents the priority for a refinery to procure the supply from farm $i$. In other words, the rank- $r$ refinery is the farm's $(r+1)$ th choice for biomass outlet. In the normal scenario (i.e., no refinery disruption), the rank-0 refinery procures the supply. If rank-0 refinery has failed, the rank- 1 refinery takes over. Such takeover continues based on the rank until all $R$ assigned refineries have failed, and then farm $i$ cannot sell its biomass and incurs a penalty cost $\phi_{i}$ due to loss of economic opportunity and adverse social impacts (Although farmers are suppliers, if the closest refinery is disrupted, they need to seek other outlets to sell their biomass. Given the transportation cost, farmers may or may not choose to ship their product to the second or more refineries. The parameter $R$ implies the trade-off between transportation cost and biomass sales profit.). For convenience, we incorporate this penalty cost into the model by defining a preinstalled virtual "emergency" facility $j=|\mathcal{J}|$ with $q_{J}=0$ that serves farm $i$ at rank $R$ with transportation cost $d_{i J}=\phi_{i}$. For the simplicity of notation, we define $\mathcal{J}^{\prime}=\mathcal{J} \bigcup\{|\mathcal{J}|\}$.

The primary decision variables $\mathbf{X}=\left\{X_{j}\right\}_{j \in \mathcal{J}}$ determine where the refineries are built; i.e.,

$$
X_{j}=\left\{\begin{array}{cc}
1, & \text { if a refinery is built at location } j ; \\
0, & \text { otherwise }
\end{array}\right.
$$

We also define two sets of auxiliary variables. The first set $\mathbf{Y}=\left\{Y_{i j r}\right\}_{i \in \mathcal{I}, j \in \mathcal{J}^{\prime}, r=0, \cdots, R}$ decides the assignment of farms to refineries (We assume that all farmers are assigned to $R$ levels of refineries, and the functioning refineries are uncapacitated.); i.e., 


$$
Y_{i j r}=\left\{\begin{array}{cc}
1, & \text { if refinery } j \text { is assigned to customer } i \text { at rank } r \\
0, & \text { otherwise. }
\end{array}\right.
$$

The second set of variables $\mathbf{P}=\left\{P_{i j r}\right\}_{i \in \mathcal{I}, j \in \mathcal{J}, r=0, \cdots, R}$ specifies the probability of farm $i$ being served by refinery $j$ at rank $r$.

The objective is to determine the optimal refinery locations and supply allocation plans to minimize the sum of facility construction cost and total expected transportation cost across all disruption scenarios. The mathematical program of reliable uncapacitated facility location (RUFL) problem can be written as follows:

$$
\begin{gathered}
\text { (RUFL) } \min _{\mathbf{X}, \mathbf{Y}, \mathbf{P}} \sum_{j \in \mathcal{J}} f_{j} X_{j}+\sum_{i \in \mathcal{I}} \sum_{j \in \mathcal{J}} \sum_{r=0}^{R} \lambda_{i} d_{i j} P_{i j r} Y_{i j r} \\
\text { s.t. } \quad \sum_{j \in \mathcal{J}} Y_{i j r}+\sum_{s=0}^{r} Y_{i J s}=1, \forall i \in \mathcal{I}, r=1, \cdots R \\
\sum_{r=0}^{R-1} Y_{i j r} \leq X_{j}, \forall i \in \mathcal{I}, j \in \mathcal{J} \\
\sum_{r=0}^{R} Y_{i J r}=1, \forall i \in \mathcal{I} \\
P_{i j 0}=1-q_{j}, \forall i \in \mathcal{I}, j \in \mathcal{J}^{\prime} \\
P_{i j r}=\left(1-q_{j}\right) \sum_{k \in \mathcal{J}} \frac{q_{k}}{1-q_{k}} P_{i k(r-1)} Y_{i k(r-1)}, \forall i \in \mathcal{I}, j \in \mathcal{J}^{\prime}, r=0,1, \cdots R \\
X_{j} \in\{0,1\}, \forall j \in \mathcal{J} \\
Y_{i j r} \in\{0,1\}, \forall i \in \mathcal{I}, j \in \mathcal{J}^{\prime}, r=0,1, \cdots R
\end{gathered}
$$

This model can be solved by a customized Lagrangian relaxation (LR) algorithm [26]. The basic idea is to relax constraints and add them into the objective with multipliers $\mu=\left\{\mu_{i j}\right\}$. The relaxed RUFL model (RRUFL) is as follows:

$$
\begin{gathered}
\text { (RRUFL) } \min _{\mathbf{X}, \mathbf{Y}, \mathbf{P}} \sum_{j \in \mathcal{J}}\left(f_{j}-\sum_{i \in \mathcal{I}} \mu_{i j}\right) X_{j} \\
+\sum_{i \in \mathcal{I}} \sum_{j \in \mathcal{J}} \sum_{r=0}^{R}\left(\lambda_{i} d_{i j} P_{i j r}+\mu_{i j}\right) Y_{i j r}+\sum_{i \in \mathcal{I}} \sum_{r=0}^{R} \lambda_{i} d_{i J} P_{i J r} Y_{i J r}
\end{gathered}
$$

s.t. Equations (4), (5)-(10).

It can be shown that the RRUFL model can be simplified by approximating variables $\left\{P_{i j r}\right\}$ into some fixed values. Let $j_{0}, j_{1}, \cdots, j_{|\mathcal{J}|-1}$ be an ordering of all candidate locations such that $q_{j_{0}} \leq q_{j_{1}} \leq \cdots \leq q_{j_{|\mathcal{|}|-1}}$. Define $\beta_{r}=\prod_{k=0}^{r-1} q_{j k}$, and replace the variable probability $P_{i j r}$ with $\left(1-q_{j}\right) \beta_{r}$. After this approximation, the problem can be solved in linear time and provides a lower bound of the original objective (but not necessarily feasible). Then the solution of $\mathbf{X}$ is plugged back into model RUFL, and with fixed $\mathbf{X}$ values the original RUFL can be easily solved to obtain a feasible solution (or an upper bound). Note that the true optimum is always between the two bounds. After obtaining these two bounds, the LR algorithm updates multipliers $\mu$ iteratively using the standard subgradient method [33]. In many cases, LR can close the optimality gap between the lower and upper bounds and 
hence yield the exact optimal solutions after a few iterations. In case a non-zero residual gap is present, the LR algorithm can be embedded into a branch and bound (BB) procedure to effectively close the gap.

\subsection{Continuous Approximation Model}

\subsubsection{The Continuous Approximation Formulation}

References [34,35] proposed a continuous approximation (CA) method to efficiently solve a variety of logistics problems in a continuous setting. In our context, we consider a 2-dimensional space $S \subset \mathbb{R}^{2}$, where the construction cost $f$, supply density $\lambda$, penalty cost $\phi$, and disruption probability $q$ are all continuous functions of point $x \in S$. Further we assume transporting each bushel of corn for a unit distance incurs an annual cost $c$. The CA approach looks for the optimal continuous facility density, $\gamma^{*}(x)$, or equivalently, the optimal influence area size of a facility, $A^{*}(x)=\left[\gamma^{*}(x)\right]^{-1}$, that minimizes the cost per unit of area $z(A(x), x)$ near location $x$. For the reliable uncapacitated facility location problem, [26] gave the following closed-form approximation of $z(A(x), x)$; i.e.,

$$
z(A(x), x) \approx \frac{f(x)}{A(x)}+\phi(x) \lambda(x)[q(x)]^{R}+c \lambda(x) G(R, q(x)) \sqrt{A(x)}
$$

Here, $G(R, q) \sqrt{A(x)}$ can be related to the expected travel cost in a homogeneous plane $\mathbb{R}^{2}$ (i.e., where all parameters $f, \lambda, \phi$ and $q$ are independent of $x$ ). A close approximation of $G(R, q)$ is given by [27]:

$$
G(R, q) \approx \frac{2}{3 \sqrt{\pi}} \sum_{r=0}^{R}(1-q) q^{r}\left[(r+1)^{3 / 2}-r^{3 / 2}\right]
$$

For notation simplicity, we omit argument $x$ from now on (unless specified otherwise), and Equation (12) becomes:

$$
z=\frac{f}{A}+\phi \lambda q^{R}+c \lambda G(R, q) \sqrt{A}
$$

Given any neighborhood $x$, the optimal influence area size that minimizes Equation (14) is:

$$
A^{*} \approx\left(\frac{f}{c \lambda G(R, q)}\right)^{2 / 3}
$$

and the corresponding cost per unit area is:

$$
z^{*} \approx 2 f^{1 / 3}(c \lambda G(R, q))^{2 / 3}+\phi \lambda q^{R}
$$

Intuitively, the optimal total costs $Z^{*}$ and the optimal number of facilities $n^{*}$ are the integrations of $z^{*}$ and $\gamma^{*}$ on $S$ :

$$
\begin{gathered}
Z^{*}=\int_{S} z^{*} d x \\
n^{*}=\int_{S} \gamma^{*} d x=\int_{S}\left(A^{*}\right)^{-1} d x
\end{gathered}
$$




\subsubsection{Discrete Data and Location Design}

In real-world applications, the CA approach needs to address several challenges. First, while the CA output $\gamma^{*}$ (e.g., facility density) has been shown repeatedly to provide very good estimates of the exact optimal solution, it is not yet the actual discrete location design that can be readily implemented. Second, the input data such as $f, \lambda, \phi$ and $q$ may be defined (or available) only at a subset of discrete points $\left\{x_{k}: k \in \mathcal{K}\right\}$ in $S$ (e.g., via spatial aggregation or sampling). These discrete data must be transformed into continuous functions to be used as an input to the CA approach.

To obtain near-optimum implementable solutions from the CA output, Ouyang and Daganzo [16] proposed a disk model to convert the continuous facility density function $\gamma^{*}$ into a set of discrete locations. After $n^{*}$ is estimated, the algorithm finds a set of $n^{*}$ round disks which cover most of $S$. A disk centered at $x_{0}$ has size $A^{*}\left(x_{0}\right)$. The locations of disk centers are determined by exerting forces to disks and iteratively adjusting their positions. Finally the disk centers are relocated to their nearest discrete candidate locations as the built facilities. Interested readers are referred to [16,36] for more details.

If the input data is discrete, we further implement a variant of the disk model [37] that (i) converts discrete data into continuous functions to calculate $A^{*}$; and (ii) obtains a discrete feasible solution. A slightly different conversion algorithm is proposed for (ii) compared with [16], because in many cases a disk covers multiple supply points. The continuous functions at $x_{0}$ is dynamically updated based on information of all data points covered by the disk. Interested readers are referred to [37] for more details.

\section{Data Sources}

This section describes the refinery infrastructure design case study for Illinois in the year of 2022 . As one of the leading states in corn production in the U.S., Illinois has been experiencing rapid bio-ethanol production infrastructure development. It becomes a major challenge to design a refinery facility system that efficiently collects biomass supply in the region and properly responds to unexpected infrastructure disruptions. We apply both discrete and continuous reliable facility location modeling techniques to tackle this problem. In this case study, county level biomass supply and refinery locations in the Illinois network are considered. The preparation of input data is discussed in the rest of the section. Since we consider an annual-equivalent static problem, for simplicity, interest rates for all prorated cost components are assumed to be zero.

Generally candidate biorefinery locations are selected based on access to major transportation networks and water availability [14,38]. In Illinois, surface water and aquifers are widely available, and the interstate and local highways are easy to access in most counties [4]. Therefore we assume that every centroid of the $|\mathcal{J}|=102$ Illinois counties is a candidate refinery location, as shown in blue empty dots in Figure 1a. According to the data from Renewable Fuels Association [39], there are 15 existing refineries in 12 different counties in the State of Illinois as shown in blue solid dots in Figure 1a. In this study, we assume that each newly built refinery costs $f_{j}=\$ 4,134,965$ per year. This number is calculated based on APEC (2010)'s estimation of a fixed cost of $\$ 65,562,227$ for a refinery with a production capacity of 50 million gallons per year and a 15 year life span. 


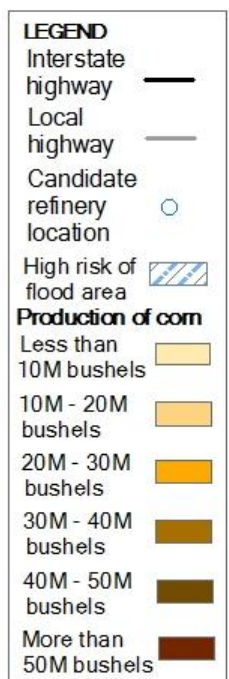

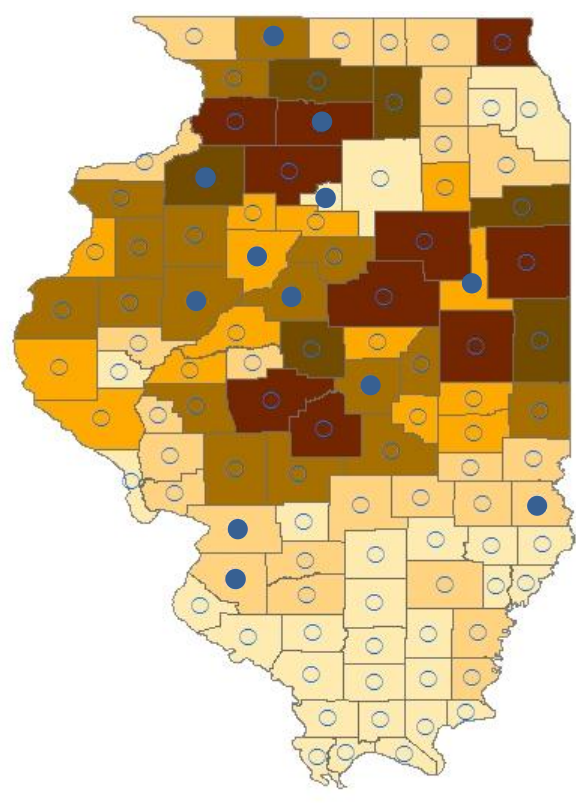

(a)

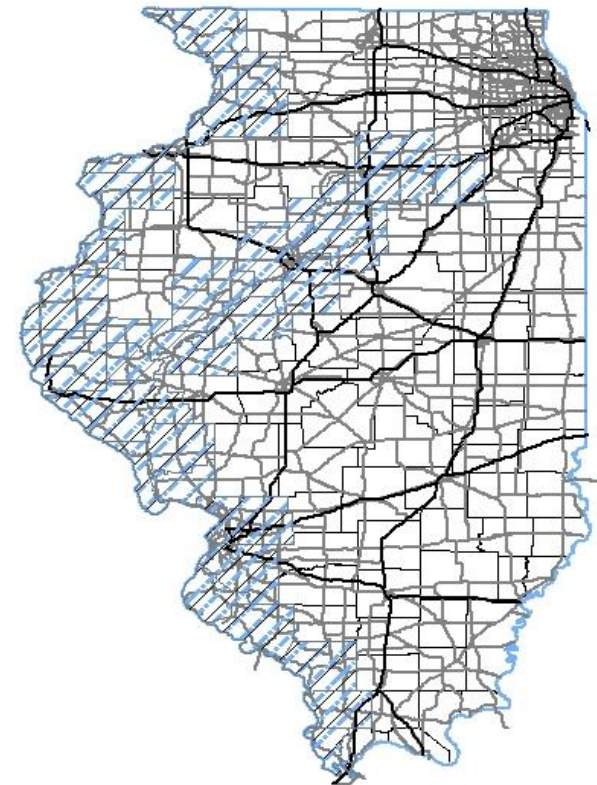

(b)

Figure 1. Maps for the Illinois case study. (a) Projected corn production projection levels, existing and candidate refinery locations in year 2022; (b) Highway network and flood-prone counties.

Each county in Illinois is also an aggregated biomass supply region, and thus $\mathcal{I}=\mathcal{J}$. The annual production level is about 9 billion gallons in 2008 and it steadily increases each year. The 2012 county level corn production data for the State of Illinois is retrieved from National Agricultural Statistics Service Database of the U.S. Department of Agriculture [40]. To estimation the county level biomass supply in Illinois in the year 2022, two scenarios are considered: (i) we projected 2022 corn biomass supply $\lambda_{i}$ based on 2012 production data and assuming a 3\% annual growth factor and $30 \%$ share of corn for ethanol use [41]; (ii) in the second scenario, we projected the 2022 corn biomass supply quantity $\lambda_{i}$ in each county based on mandatory ethanol demand in 2022 and fixed proportions of county level production in 2012. According to the 2007 EPA mandate, the national target for ethanol production is 15 billion gallons from corn in 2022 [1]. Furthermore, we assume that Illinois produces $9 \%$ of the nation's mandatory ethanol demand and one bushel of corn yields about 2.8 gallons of ethanol [42].

In the US, most biomass is shipped by truck because it is shown to be more economical for short-distance transportation (e.g., no more than $200 \mathrm{~km}$ ) than other transportation modes (such as rail and barge) (In very limited cases such as long-distance transshipment, rail and barge are preferred for biomass shipment.). Thus a refinery of an economical size would attract biomass supply from within an economical truck travel distance. We used the approach proposed by Kang et al. [5] to calculate the travel distance from each biomass supply point to each candidate location along the shortest-distance path in the GIS highway network provided by the Bureau of Transportation Statistics National Transportation Atlas Database [43]. Assuming that third-party carriers are used, the cost for shipping a bushel of corn is $\$ 0.0017$ per km [44]. Since the CA model measures distances by the Euclidean metric (instead of the highway network), all Euclidean distances are inflated by a factor of 1.206 so that the average Euclidean distance is the same as the average highway distance. 
Ethanol refineries may be disrupted by site-independent hazards such as power outages, operational incidents, and labor actions. We assume that these refinery disruptions are independent of each other. In addition, Illinois is covered by a dense web of rivers (including primarily the Mississippi River and Illinois River) that pose major flooding threats to refineries in Illinois. The probability for a refinery to be caught in a flood highly depends on the location and geographical configuration (See the Flood Insurance Rate Maps (FIRMs) at http://www.fema.gov/hazard/map/firm.shtm). Therefore, we define the disruption probability as follows: $q_{j}=\bar{q}+\hat{q}_{j}$, where the site-independent component $\bar{q}$ counts for multiple common disruption hazards, and the site-dependent component $\hat{q}_{j}$ represents local flooding disruption probability. The value of probability $\bar{q}$ is estimated to be 0.05 , based on refinery disruption records in California from 1997 to 2001 [30]. Furthermore, we calculate flooding probability of each county of Illinois State using the data from 1999 to 2013 obtained from FEMA [45], which range from 0\%-3\%. Figure 1b shows the flood prone counties near Mississippi River and Illinois River which in general have higher flooding probabilities than other counties. However, very limited data on disruption probabilities due to flooding are publicly accessible. We define a parameter $\sigma \geq 0$ as "flooding disruption ratio" (The flood disruption ratio $\sigma$ indicates the ratio between the duration that biorefineries are under disruption and the duration of a flood) and assume that the flood disruption probability $\hat{q}_{j}$ equals to the product of $\sigma$ and the empirical flooding probability for all $j \in J$, and we will examine a range of values for $\sigma$ in the sensitivity analysis.

We assume that a farm incurs a penalty cost after three assigned refineries have all been disrupted; i.e., $R=3$. The penalty cost value (per unit biomass supply) is approximated by the net social cost due to the corresponding ethanol supply loss [30]. Basically, refinery disruptions may lead to biomass supply loss and hence ethanol shortage. For simplicity, we assume that the ethanol price is at equilibrium in an efficient market. In case of disruptions, incremental supplies are sourced out of precautionary inventories and/or from distant sources, which would raise local ethanol price. According to the 2002 California Energy Commission report [30], the price response at the time of a disruption is almost instant. The mechanism of price rise to clear the market is in accordance with ethanol demand elasticity. In the interim after the disruption and before the price return to normal, the impact on the net social cost is contributed by a loss of consumer surplus (which is a standard measure of societal benefit from an industry). In this sense, the penalty cost used in this study can be interpreted as the net societal cost or the total loss of consumer surplus. By applying the method and empirical data in [30] and the latest price data [46], we obtain a $\$ 2.64 /$ gallon retail price of corn ethanol, which has a production rate of 135 million gallons per year across Illinois (i.e., scenario ii), and a combined price elasticity of -0.15 . Then, one gallon of unmet ethanol demand induces $\$ 3.96$ total loss of consumer surplus in the society. Therefore, the unit equivalent penalty cost is roughly $\phi_{i}=16.7 \$ /$ bushel.

\section{Numerical Results}

This section presents numerical results of the case study. All solution algorithms are implemented on a personal computer with 3.0 GHz CPU and $2 \mathrm{~GB}$ memory. The LR algorithm is coded in $\mathrm{C}++$, while the CA approach is coded in MATLAB 8.3. (For simplicity, advanced data structures such as range tree and 
KD-tree are not implemented. So it took $O(|\mathcal{K}|)$ instead of $O(\log |\mathcal{K}|)$ time to make an orthogonal range counting query or a nearest neighbor search.)

\subsection{Computational Performance}

To test the computational performance of different solution approaches for the reliable biorefinery problem, we run the following three cases for three approaches: LR, CA estimation, and CA with disk model:

Case 1: all built refineries are robust and never fail (i.e., $q_{j}=0$ for all $j$ );

Case 2: all built refineries are subject to a homogeneous disruption probability $q_{j}=\bar{q}$ for all $j$;

Case 3: all built refineries are also under the threat of flooding, so that the disruption probability $q_{j}$ is heterogeneous across location $j$.

To show the differences among the three cases, a reduced fixed cost value is used, i.e., $40 \% * f_{j}$, $\forall j \in J$, and all other parameter values are the same as introduced in Section 3. We also perform a sensitivity analysis on different levels of fixed cost in the following subsection.

The results are shown in Table 1. It can be seen that LR can obtain the near optimal solutions within half an hour. The CA model yields the estimated total costs $Z^{*}$ and the optimal number of refineries $n^{*}$ almost instantaneously, which are very close to their discrete counterparts (which is near optimal). Note that the CA estimation does not necessary yields feasible solutions, hence its objective value can be either larger or smaller than the real optima. The results of the disk model are obtained within $0.3 \mathrm{~s}$, while the gap is at most $7.5 \%$. This relatively large gap may be due to several reasons. First, the disk model is suitable for large-scale problems with slow-varying conditions. However, the scale of our problem instance is actually not extremely large (102 data points) while the spatial distribution of biomass density (i.e., production of corn) is relatively heterogeneous. It is also found that the discrete model (using LR) tends to locate facilities to counties with high corn yield and low flood probabilities, even if these locations are close to the geographical boundary. In contrast, the disk model generally is not able to do so when the location is very near the boundary. The details of the boundary issue for continuous model are discussed in Cui et al. [26]. In addition, the disk model uses the adjusted Euclidean distances, which may not be a close approximation to the real highway distances. Finally, we use highly-aggregated biomass production data and a small set of discrete candidate locations. If the biomass supply data have a higher spatial resolution or if the facilities are allowed to be located anywhere in the state, the disk model will be expected to have a better performance. The gap is actually relative gap between the best solution (upperbound) and the best lowerbound obtained by LR algorithm, i.e.,

$$
\text { Optimality gap }=(\text { upperbound }- \text { lowerbound }) / \text { lowerbound } \times 100 \%
$$


Table 1. Summary of different model solutions for different cases.

\begin{tabular}{|c|c|c|c|c|c|c|c|c|c|c|}
\hline \multirow{3}{*}{ Algorithm } & \multirow{3}{*}{ Case } & \multirow{3}{*}{$\bar{q}$} & \multirow{3}{*}{$\sigma$} & \multirow{3}{*}{$\begin{array}{c}\text { Number of } \\
\text { refineries }\end{array}$} & \multicolumn{4}{|c|}{ Expected Cost $(\$ M *)$} & \multirow{3}{*}{$\begin{array}{c}\text { Optimality Gap } \\
\text { or Error }\end{array}$} & \multirow{3}{*}{$\begin{array}{l}\text { Solution } \\
\text { Time (s) }\end{array}$} \\
\hline & & & & & \multirow{2}{*}{ Facility } & \multicolumn{2}{|c|}{ Transportation } & \multirow{2}{*}{ Total } & & \\
\hline & & & & & & Normal & Disrupted & & & \\
\hline \multirow{3}{*}{ LR } & 1 & 0 & 0 & 17 & 8.27 & 36.11 & 0.00 & 44.38 & $0 \%$ & 2135 \\
\hline & 2 & 0 & 0 & 17 & 8.27 & 34.31 & 4.59 & 47.17 & $0 \%$ & 731 \\
\hline & 3 & $5 \%$ & 1 & 17 & 8.27 & 34.05 & 5.64 & 47.97 & $2 \%$ & 990 \\
\hline \multirow{3}{*}{$\begin{array}{c}\mathrm{CA} \\
\text { (estimate) }\end{array}$} & 1 & 0 & 0 & 18 & 11.4 & 31.29 & 0 & 44.05 & $-0.7 \%$ & $<0.01$ \\
\hline & 2 & $5 \%$ & 0 & 19 & 12.3 & 29.32 & 3.88 & 46.89 & $-0.6 \%$ & $<0.01$ \\
\hline & 3 & $5 \%$ & 1 & 19 & 12.5 & 28.88 & 5.43 & 48.24 & $0.6 \%$ & $<0.01$ \\
\hline \multirow{3}{*}{$\begin{array}{c}\text { CA } \\
\text { (disk model) }\end{array}$} & 1 & 0 & 0 & 18 & 9.92 & 37.81 & 0 & 47.73 & $7.5 \%$ & 0.2 \\
\hline & 2 & $5 \%$ & 0 & 19 & 11.5 & 31.92 & 4.43 & 47.92 & $1.6 \%$ & 0.3 \\
\hline & 3 & $5 \%$ & 1 & 19 & 11.5 & 32.07 & 6.02 & 49.66 & $3.5 \%$ & 0.3 \\
\hline
\end{tabular}

Note: * Million dollars.

\subsection{Impact of Refinery Disruptions}

To understand the impact of refinery disruption on the optimal bio-ethanol supply chain design and performance, we make the case study as realistic as possible using empirical data and compare two models: (i) a deterministic model in which biorefinery disruption risks are not considered in the optimal solution; (ii) the reliable model as presented in Section 2. Both models are solved by the LR approach under $3 \%$ gap tolerance and $1000 \mathrm{~s}$ time limit. Optimal solutions of both models for a scenario with $\bar{q}=0.15, \sigma=1$ are applied to evaluate itemized costs, as shown in Table 2 and Figure 2.

Table 2. Comparison between the deterministic and reliable model.

\begin{tabular}{|c|c|c|c|c|c|c|c|c|}
\hline \multirow{3}{*}{ Models } & \multirow{3}{*}{$\begin{array}{c}\text { Number } \\
\text { of New } \\
\text { Refineries }\end{array}$} & \multicolumn{5}{|c|}{$\begin{array}{c}\text { Expected Cost (The Total Cost Includes Only Refinery Capital } \\
\text { Cost and Biomass Transportation Cost) }\end{array}$} & \multicolumn{2}{|c|}{ Normal Cost } \\
\hline & & \multirow{2}{*}{$\begin{array}{c}\text { Facility } \\
(\$ M)\end{array}$} & \multicolumn{2}{|c|}{ Transportation $(\$ M)$} & \multirow{2}{*}{$\begin{array}{l}\text { Total } \\
(\$ M)\end{array}$} & \multirow{2}{*}{$\begin{array}{l}\text { Unit Cost * } \\
(\$ / \text { Gallon })\end{array}$} & \multirow{2}{*}{$\begin{array}{l}\text { Total } \\
(\$ M)\end{array}$} & \multirow{2}{*}{$\begin{array}{l}\text { Unit Cost } \\
\text { (\$/Gallon }\end{array}$} \\
\hline & & & Normal & Disrupted & & & & \\
\hline Deterministic Model & 5 & 8.27 & 30.44 & 43.15 & 81.86 & 0.17 & 44.38 & 0.089 \\
\hline Reliable Model & 6 & 9.92 & 29.49 & 42.28 & 81.69 & 0.16 & 44.90 & 0.090 \\
\hline
\end{tabular}

* The unit cost is calculated by dividing the total cost by the total amount of corn used. The lost corn due to disruption (that are shipped to the "emergency facility") is not considered. Besides, other major costs that are exogenous to our optimization model are not included, such as feedstock procurement and biomass conversion.

Table 2 compares optimal costs of the two models. The reliable model reduces the transportation cost in both normal and disrupted scenarios, and also the total system cost by building one more new refinery. Specifically, when disruption risk is present, if we use the refinery deployment from the deterministic result, the expected transportation cost across disrupted cases will increase by $2.5 \%$ (although the total costs increase only by $0.21 \%$ due to the higher fixed facility investments). Figure 2 shows the impact of disruption risks on the optimal facility deployment. The reliable model selects one more location than the deterministic model (i.e., in county Warren to back up the other relocated refinery as shown in Figure 2b). It indicates that when there are disruption risks, especially under high probability, it often necessary to build more refineries as back up facilities to save total system cost considering disruption 
scenarios. Therefore, facility disruption risks should not be ignored in supply network design, especially when the disruption consequences are devastating.

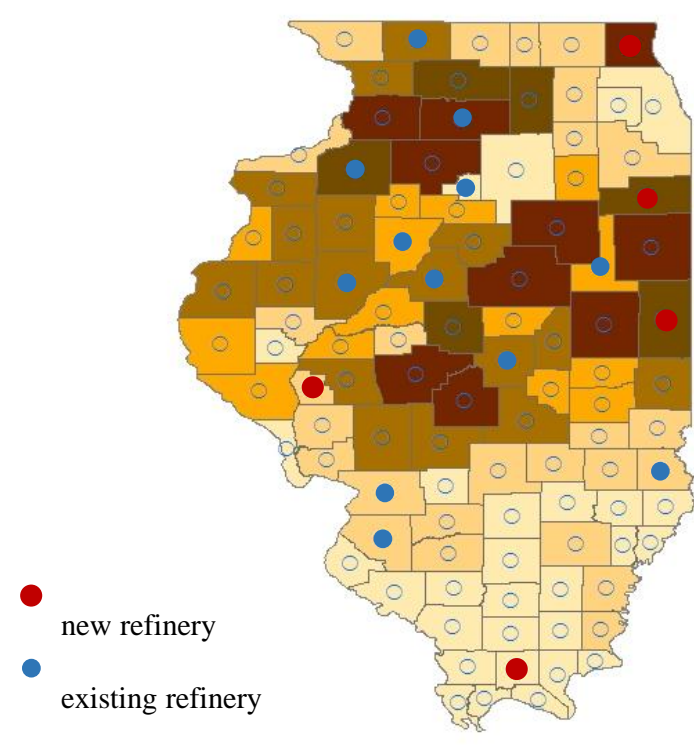

(a)

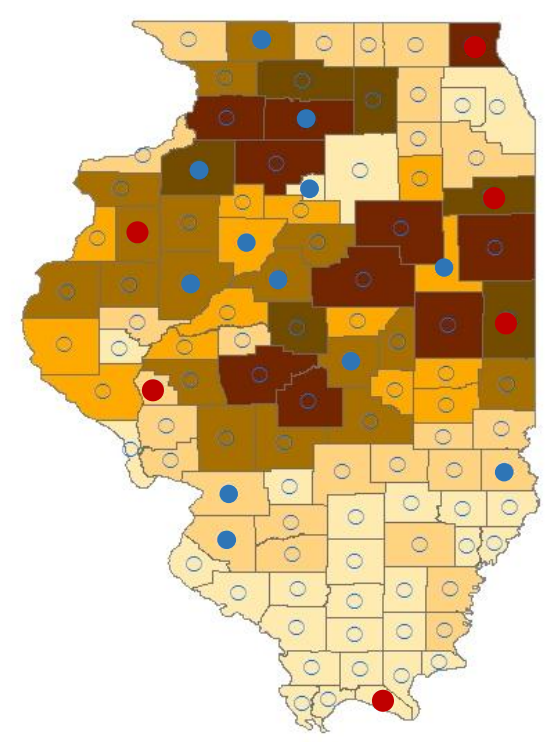

(b)

Figure 2. Optimal refinery locations from the deterministic and reliable models. (a) deterministic model; (b) reliable model.

We also evaluate the impacts of disruption uncertainties and reliable design on the total and unit costs for producing bioethanol, as revealed in the 6th to 9th columns in Table 2. Obviously, disruption raises the expected bioethanol total cost by $78 \%$ under reliable design and $91 \%$ under deterministic design, respectively. Such a huge impact is resulted from the high disruption probability assumed in this case, which highlights the fact that disruption uncertainties should not be neglected in disaster-prone areas. In other words, the system is more vulnerable to disruption if refineries are deployed under deterministic design. Note that the deterministic model saves total and unit costs only in the normal scenario (i.e., disruption probabilities are zero everywhere) as shown in the last two columns, which is not true for the expected cost.

In addition, the reliable design model results in lower unit cost than the deterministic one, which attributes to not only the reduced total cost (i.e., column 6 in Table 2), but also increased supply chain throughput (e.g., less unused biomass).

\subsection{Sensitivity Analysis}

The key trade-off that the reliable biofuel supply chain problem needs to address is between the cost for building backup refineries and the excessive transportation/penalty cost in disrupted scenarios. It essentially depends on the relative ratio between the fixed cost and transportation or penalty cost that matters. To study this impact, we did a sensitivity analysis first on the fixed cost with all other cost parameter values fixed (i.e., the same as the benchmark case in Section 4.2). The results are summarized in Table 3. Given the existing refineries, no new locations are selected to build refineries under the current fixed cost. Only when the fixed cost is reduced below $80 \%$ (e.g., due to future technology 
improvement), building new refineries starts to become optimal. As we expect, higher fixed cost results in fewer refineries to be built. However, the total fixed cost also depends on the number of refineries, and thus it may actually shrink even though the individual fixed cost gets higher.

Table 3. Sensitivity of optimal solutions to fixed cost.

\begin{tabular}{|c|c|c|c|c|c|}
\hline \multirow{3}{*}{ Fixed Cost } & \multirow{3}{*}{$\begin{array}{c}\text { Number of } \\
\text { New Refineries }\end{array}$} & \multicolumn{4}{|c|}{ Expected Cost (\$M) } \\
\hline & & \multirow{2}{*}{ Facility } & \multicolumn{2}{|c|}{ Transportation } & \multirow{2}{*}{ Total } \\
\hline & & & Normal & Disrupted & \\
\hline $20 \%$ & 13 & 10.75 & 25.53 & 4.41 & 40.68 \\
\hline $40 \%$ & 5 & 8.27 & 34.18 & 5.10 & 47.55 \\
\hline $60 \%$ & 3 & 7.44 & 37.65 & 5.54 & 50.63 \\
\hline $80 \%$ & 1 & 3.31 & 43.31 & 5.83 & 52.44 \\
\hline $100 \%$ & 0 & 0.00 & 47.18 & 5.85 & 53.02 \\
\hline
\end{tabular}

In our case study, the refinery disruption probability $\bar{q}$ is estimated from an empirical study in California. Those values may not be suitable for Illinois due to geographical, climate, and political differences. Hence, we conduct sensitivity analysis over a range of possible values for $\bar{q}$, in which again we used $40 \%$ of the current fixed cost value to show how the optimal number of installations change with $\bar{q}$. The results are summarized in Table 4 . We see that the refinery number slightly increases with $\bar{q}$ (however, the refinery deployment may change considerably). The total cost increases with $\bar{q}$ dramatically for larger $\bar{q}$. Also note that the normal transportation cost decreases with $\bar{q}$ while the transportation cost under disrupted scenarios increase with it. Besides, the normal transportation cost decreases slightly as the disruption probability increases, this is because the chance of the normal scenario decreases (and the disruption scenarios that yield higher transportation costs are more likely).

Table 4. Sensitivity of optimal solutions to site independent probability $\bar{q}$.

\begin{tabular}{cccccc}
\hline \multirow{2}{*}{$\begin{array}{c}\text { Site-Independent } \\
\text { Probability }\end{array}$} & $\begin{array}{c}\text { Number of } \\
\text { New Refineries }\end{array}$ & Facility & \multicolumn{4}{c}{ Expected Cost (\$M) } \\
\cline { 4 - 5 } & 5 & 8.27 & Normsportation & Disrupted & \\
\hline 0.01 & 5 & 8.27 & 34.18 & 5.10 & 47.55 \\
0.05 & 5 & 8.27 & 32.37 & 16.58 & 57.23 \\
0.10 & 5 & 8.27 & 30.57 & 40.85 & 79.69 \\
0.15 & 6 & 9.92 & 29.33 & 46.89 & 86.14 \\
0.16 & 6 & 9.92 & 28.89 & 54.58 & 93.39 \\
0.17 & 7 & 11.58 & 27.57 & 62.33 & 101.47 \\
0.18 & 6 & 9.92 & 28.21 & 72.64 & 110.78 \\
0.19 & 7 & 11.58 & 26.98 & 82.04 & 120.60 \\
0.20 & & & & & \\
\hline
\end{tabular}

As we mentioned earlier, accurate disruption probabilities due to flooding are difficult to acquire. We further examine a range of flood disruption ratio $\sigma$ values in the sensitivity analysis under $40 \%$ and $100 \%$ of the current fixed cost value and $\bar{q}=5 \%, 15 \%$, and $20 \%$. Table 5 shows that the optimal solution is not sensitive to the flood disruption ratio unless when the site independent probability $\bar{q}$ is high, e.g., case 6-8. This is because the optimal biorefinery locations (including the 12 existing ones) are 
mostly in the counties with low flood probability. Even with high flood disruption ratio, flooding impact alone on refinery location selection is small. Again, both the transportation cost due to disruption and the total cost increase with $\sigma$ (and thus $\hat{q}$ ).

Table 5. Sensitivity of optimal solutions to flood disruption ratio $\sigma$.

\begin{tabular}{|c|c|c|c|c|c|c|c|c|}
\hline \multirow{3}{*}{$\begin{array}{c}\text { Case } \\
\text { Number }\end{array}$} & \multirow{3}{*}{$\begin{array}{c}\text { Fixed } \\
\text { Cost }\end{array}$} & \multirow{3}{*}{$\begin{array}{c}\text { Site } \\
\text { Independent } \\
\text { Probability } \bar{q}\end{array}$} & \multirow{3}{*}{$\begin{array}{c}\text { Flood } \\
\text { Disruption } \\
\text { Ratio } \sigma \\
\end{array}$} & \multirow{3}{*}{$\begin{array}{c}\text { Number } \\
\text { of New } \\
\text { Refineries }\end{array}$} & \multicolumn{4}{|c|}{ Expected Cost (\$M) } \\
\hline & & & & & \multirow{2}{*}{ Facility } & \multicolumn{2}{|c|}{ Transportation } & \multirow{2}{*}{ Total } \\
\hline & & & & & & Normal & Disrupted & \\
\hline 1 & & & 0.50 & 5 & 8.27 & 34.18 & 5.10 & 47.55 \\
\hline 2 & & 0.05 & 1 & 5 & 8.27 & 34.05 & 5.64 & 47.97 \\
\hline 3 & & & 2 & 5 & 8.27 & 33.80 & 6.83 & 48.90 \\
\hline 4 & & & 0.5 & 5 & 8.27 & 30.57 & 40.85 & 79.69 \\
\hline 5 & $40 \%$ & 0.15 & 1 & 6 & 9.92 & 29.49 & 42.28 & 81.69 \\
\hline 6 & & & 2 & 7 & 11.58 & 28.32 & 45.48 & 85.37 \\
\hline 7 & & & 0.5 & 7 & 11.58 & 26.87 & 81.93 & 120.39 \\
\hline 8 & & 0.20 & 1 & 6 & 9.92 & 27.72 & 86.52 & 124.16 \\
\hline 9 & & & 2 & 7 & 11.58 & 26.55 & 91.71 & 129.84 \\
\hline 10 & & & 0.5 & $0 *$ & 0.00 & 47.18 & 5.85 & 53.02 \\
\hline 11 & & 0.05 & 1 & 0 & 0.00 & 47.01 & 6.36 & 53.37 \\
\hline 12 & & & 2 & 0 & 0.00 & 46.67 & 7.44 & 54.12 \\
\hline 13 & & & 0.5 & 0 & 0.00 & 42.19 & 42.82 & 85.01 \\
\hline 14 & $100 \%$ & 0.15 & 1 & 0 & 0.00 & 42.03 & 44.83 & 86.86 \\
\hline 15 & & & 2 & 0 & 0.00 & 41.69 & 49.01 & 90.70 \\
\hline 16 & & & 0.5 & 0 & 0.00 & 39.70 & 86.48 & 126.19 \\
\hline 17 & & 0.20 & 1 & 0 & 0.00 & 39.53 & 89.78 & 129.31 \\
\hline 18 & & & 2 & 0 & 0.00 & 39.20 & 96.59 & 135.78 \\
\hline
\end{tabular}

* The zero facility cost in Table 5 means that no new refinery location is selected, and the system only uses the 12 existing refineries, therefore, there is still transportation cost.

In summary, as the disruption probabilities increase, the expected transportation cost increases significantly, while the normal transportation cost reduces (which indicates that the amount of biomass supply processed by its 1 st choice outlet, i.e., its nearest refinery, reduces because of disruption). As a result, the total cost rises considerably. This strongly hints that facility disruption risks, if present, should not be ignored in cost analysis.

\section{Conclusions}

This paper studies the supply chain network design problem for the ongoing biorefinery infrastructure planning in the State of Illinois. Reliable facility location design models are applied to determine optimal refinery locations that can hedge against the risk of refinery operation disruptions and the consequence of enormous social disbenefits. Both a discrete and a continuous version of the reliable location models have been implemented. The data sets used in this case study are extracted from the Illinois biomass supply databases and various technical reports on refinery disruptions.

We found that both reliable location models can efficiently solve the supply chain design problem while each offers certain unique advantages. For example, the discrete model solution achieves better 
optimality while the continuum model has superior computational tractability. From our numerical experiments, we find that the optimal refinery locations tend to be at places with high biomass supply and low disruption probability, and the proposed reliable model selects more refinery locations to backup other refineries under disruption.

The numerical results show that as the disruption risk increases, it is worth investing more biofuel refineries to ensure that the service capacity remains sufficient despite facility disruptions. As individual refinery opening cost increases, the total number of built facilities drops but the total facility investment cost rises. The transportation cost under the normal scenario (i.e., no disruption) is sensitive to the individual refinery opening cost, whereas that of the disrupted transportation cost is not. As more refineries are disrupted, it is beneficial to build new refineries to slow down the increase of the transportation cost under disruption scenarios. However, if the individual refinery opening cost gets too high, this benefit may be completely compromised by the overly high facility investment. This implies that new biofuel infrastructure investment needs to be scrutinized carefully by weighing the benefit and cost, particularly when disruption risks are present, and the planner needs to avoid investing in the backup capabilities (from newly built refineries) due to their excessive costs.

Future research may be conducted in a few directions. This study assumes that the future biomass supply (and demand) is deterministic and known. However, uncertainties are prevailing in corn productions depending on unpredictable factors such as weather and economy. Our work can be also extended to also address supply outage or production congestion caused by unexpected demand fluctuations. Furthermore, since there is no commercial level production yet of advanced cellulosic biomass from dedicated energy crops, the case study does not include the cellulosic based ethanol supply chain. The optimal supply chain design scenarios are likely to be different for 2022 when such information becomes available. Furthermore, the current models assume that the capacities of all functioning refineries are never reached. This may not be realistic, especially under facility disruptions, when surviving refineries have to face reallocated biomass supply. Besides, considering the high transportation cost of shipping bulk biomass, if the primary refinery fails, it may not be profitable for a farmer to ship biomass to refineries that are farther away, i.e., $R>1$. A reasonable value of $R$ needs to be estimated to reflect such trade-off between the transportation cost and biomass price at the time of decision making. This issue should be addressed in future studies. Finally, it would be also interesting to investigate the impacts of the disruptions on the consumers from both behavioral (e.g., risk-taking behaviors) and economic (e.g., gas price fluctuations) points of view.

\section{Acknowledgments}

The first author would like to thank M.S. student Xinlu Liu (University of Illinois) for helping collect part of the data used in this study. This research was supported in part by the U.S. National Science Foundation via Grants The Civil, Mechanical and Manufacturing Innovation (CMMI) \#0748067, Emerging Frontiers in Research and Innovation-Resilient and Sustainable Infrastructure (EFRI-RESIN) \#0835982 and CMMI \#1234936, by the Mississippi Department of Transportation via Grant \#SPR-1(66)/106461-179000 and by the National Center for Intermodal Transportation for Economic Competitiveness via Grant \#DTRT12-G-UTC14. 


\section{Author Contributions}

Yun Bai, Xiaopeng Li, Fan Peng, Xin Wang, and Yanfeng Ouyang all contributed to developing the methodology, designing the numerical experiments, and writing the paper; Xiaopeng Li, Fan Peng, and Xin Wang coded the programming scripts; Yun Bai and Xiaopeng Li analyzed the data; Yun Bai and Xin Wang performed the numerical experiments.

\section{Conflicts of Interest}

The authors declare no conflict of interest.

\section{References}

1. U.S. EPA (United States Environmental Protection Agency). Renewable Fuel Standard Implementation, 2013. Available online: http://www.epa.gov/OTAQ/renewablefuels/index.htm (accessed on 1 May 2014).

2. BBI International (Biofuels Canada). Biofuel Costs, Technologies and Economics in APEC Economies (Final Report); APEC\#210-RE-01.21; APEC Energy Working Group (EWG): Singapore, 2010.

3. Jacobson, J.J.; Searcy, E.; Muth, D.; Wilkerson, E.; Sokhansanj, S.; Jenkins, B.; Nelson, R. Sustainable Biomass Supply Systems; INL/CON-09-15568; Idaho National Laboratory: Idaho Falls, ID, USA, 2009.

4. Tursun, D.; Kang, S.; Onal, H.; Ouyang, Y.; Scheffran, J. Optimum biorefinery locations and transportation network for the future biofuels industry in Illinois. In Proceedings of Transition to a Bioeconomy: Environmental and Rural Development Impacts, the Farm Foundation/USDA Conference, St. Louis, MO, USA, 15-16 October 2008.

5. Kang, S.; Önal, H.; Ouyang, Y.; Scheffran, J.; Tursun, D. Optimizing the biofuels infrastructure: Transportation networks and biorefinery locations in Illinois. In Handbook of Bioenergy Economics and Policy; Khanna, M., Scheffran, J., Zilberman, D., Eds.; Springer: New York, NY, USA, 2009; Volume 33, pp. 151-173.

6. Bai, Y.; Hwang, T.; Kang, S.; Ouyang, Y. Biofuel refinery location and transportation planning under traffic congestion. Transp. Res. B Methodol. 2011, 45, 162-175.

7. Ekşioğlu, S.D.; Acharya, A.; Leightley, L.E.; Arora, S. Analyzing the design and management of biomass-to-biorefinery supply chain. Comput. Ind. Eng. 2009, 57, 1342-1352.

8. Sokhansanj, S.; Kumar, A.; Turhollow, A.F. Development and implementation of integrated biomass supply analysis and logistics model (IBSAL). Biomass Bioenergy 2006, 30, 838-847.

9. Western Bioenergy Assessment Team. Strategic Assessment of Bioenergy Development in the West-spatial Analysis and Supply Curve Development; The University of California: Davis, IL, USA, 2008.

10. Eathington, L.; Swenson, D.A. Dude, Where's my corn? Constraints on the location of ethanol production in the Corn Belt. In Proceedings of the Annual Meeting of the Southern Regional Science Association, Charleston, SC, USA, 29-31 Match 2007. 
11. Panichelli, L.; Gnansounou, E. GIS-based approach for defining bioenergy facilities location: A case study in northern Spain based on marginal delivery costs and resources competition between facilities. Biomass Bioenergy 2008, 32, 289-300.

12. Kumar, A.; Sokhansanj, S.; Flynn, P.C. Development of a multicriteria assessment model for ranking biomass feedstock collection and transportation systems. Appl. Biochem. Biotechnol. 2006, 129, 71-87.

13. Mahmudi, H.; Flynn, P.C. Rail vs. truck transport of biomass. Appl. Biochem. Biotechnol. 2006, 129, 88-103.

14. Searcy, E.; Flynn, P.; Ghafoori, E.; Kumar, A. The relative cost of biomass energy transport. Appl. Biochem. Biotechnol. 2007, 137-140, 639-652.

15. Daskin, M. Network and Discrete Location: Models, Algorithms, and Applications; John Wiley: New York, NY, USA, 1995.

16. Ouyang, Y.; Daganzo, C.F. Discretization and validation of the continuum approximation scheme for terminal system design. Transp. Sci. 2006, 40, 89-98.

17. Newell, G.F. Scheduling, location, transportation, and continuum mechanics; some simple approximations to optimization problems. SIAM J. Appl. Math. 1973, 25, 346-360.

18. Langevin, A.; Mbaraga, P.; Campbell, J.F. Continuous approximation models in freight distribution: An overview. Transp. Res. B Methodol. 1996, 30, 163-188.

19. Daganzo, C.F. Logistics Systems Analysis, 4th ed.; Springer-Verlag: Heidelberg, Germany, 2005.

20. Daskin, M.S. Application of an expected covering model to emergency medical service system design. Decis. Sci. 1982, 13, 416-439.

21. Daskin, M.S. Maximum expected covering location model: formulation, properties and heuristic solution. Transp. Sci. 1983, 17, 48-70.

22. Ball, M.O.; Lin, F.L. A reliability model applied to emergency service vehicle location. Oper. Res. 1993, 41, 18-36.

23. ReVelle, C.; Hogan, K. The maximum availability location problem. Transp. Sci. 1989, 23, 192-200.

24. Batta, R.; Dolan, J.M.; Krishnamurthy, N.N. The maximal expected covering location problem: Revisited. Transp. Sci. 1989, 23, 277-287.

25. Snyder, L.V.; Daskin, M.S. Reliability models for facility location: The expected failure cost case. Transp. Sci. 2005, 39, 400-416.

26. Cui, T.; Ouyang, Y.; Shen, Z.M. Reliable facility location under the risk of disruptions. Oper. Res. 2010, 58, 998-1011.

27. Li, X.; Ouyang, Y. A continuum approximation approach to reliable facility location design under correlated probabilistic disruptions. Transp. Res. B Methodol. 2010, 44, 535-548.

28. Schill, S.R. Flooding Impacts Iowa Ethanol, 2008. Available online: http://www.ethanolproducer.com/ article.jsp?article_id=4333 (accessed on 30 January 2010).

29. Ford, A. Simulating the impacts of a strategic fuels reserve in California. Energy Policy 2005, 33, 483-498.

30. Finizza, A. Economic Benefits of Mitigating Refinery Disruptions, Study Conducted by AJF Consulting; CEC Report P600-02-018D; California Energy Commission: Dana Point, CA, USA, 2002. 
31. Marufuzzaman, M.; Eksioglu, S.D.; Li, X.; Wang, J. Analyzing the impact of intermodal-related risk to the design and management of biofuel supply chain. Transp. Res. E Logist. Transp. Rev. 2014, 69, 122-145.

32. Li, X.; Ouyang, Y. Reliable traffic surveillance sensor deployment under probabilistic disruptions and generalized surveillance effectiveness measures. Oper. Res. 2012, 60, 1183-1198.

33. Fisher, M.L. The Lagrangian relaxation method for solving integer programming problems. Manag. Sci. 1981, 27, 1-18.

34. Newell, G.F. Dispatching policies for a transportation route. Transp. Sci. 1971, 5, 91.

35. Daganzo, C.F.; Newell, G.F. Configuration of physical distribution networks. Networks 1986, 16, 113-132.

36. Ouyang, Y. Design of vehicle routing zones for large-scale distribution systems. Transp. Res. B 2007, 41, 1079-1093.

37. Peng, F.; Wang, X.; Ouyang, Y. Approximation of discrete spatial data for continuous facility location design. Integr. Comput. Aided Eng. 2014, 21, 311-320.

38. Peluso, T.; Baker, L.; Thomassin, P.J. The siting of ethanol plants in Quebec. Can. J. Reg. Sci. 1998, 21, 73-86.

39. RFA. Renewable Fuels Association: biorefinery locations, 2014. Available online: http://www.ethanolrfa.org/bio-refinery-locations/ (accessed on 10 July 2014).

40. U.S. Department of Agriculture-National Agricultural Statistics Service, 2014. Available online: http://quickstats.nass.usda.gov/ (accessed on 1 June 2014).

41. Taylor, D.R.; Koo, W.W. 2013 Outlook of the U.S. and World Corn and Soybean Industries, 2012-2022; Economics Report No. 713. Available online: http://ageconsearch.umn.edu/bitstream/ 157670/2/AAE713.pdf (accessed on 4 March 2014).

42. NEO: Nebraska Energy Office. Ethanol Facilities' Capacity by State, 2014. Available online: http://www.neo.ne.gov/statshtml/121.htm (accessed on 11 July 2014).

43. RITA: Research and Innovative Technology Administration. National Transportation Atlas Database, 2014. Available online: http://www.rita.dot.gov/bts/sites/rita.dot.gov.bts/files/publications/ national_transportation_atlas_database/2014/index.html (accessed on 10 July 2014).

44. Gonzales, D.; Searcy, E.M.; Ekşioğlu, S.D. Cost analysis for high-volume and long-haul transportation of densified biomass feedstock. Transp. Res. A Policy Pract. 2013, 49, 48-61.

45. FEMA: Federal Emergency Management Agency. Flood Hazard Data, 2013. Available online: http://www.fema.gov/ (accessed on 4 March 2014).

46. USDA-ERS: U.S. Department of Agriculture-Economic Research Service. Fuel ethanol, corn and gasoline prices, by month, 2014. Available online: http://www.ers.usda.gov/datafiles/US_Bioenergy/ Prices/table14.xls/ (accessed on 10 July 2014).

(C) 2015 by the authors; licensee MDPI, Basel, Switzerland. This article is an open access article distributed under the terms and conditions of the Creative Commons Attribution license (http://creativecommons.org/licenses/by/4.0/). 